\title{
PID vs LQR controller for tilt rotor airplane
}

\author{
Aoued Houari ${ }^{1}$, Imine Bachir ${ }^{2}$, Della Krachai Mohamed ${ }^{3}$, Mohamed Kara Mohamed ${ }^{4}$ \\ ${ }^{1}$ Department of Electronics, University of Sciences and Technology of Oran MB, Algeria \\ ${ }^{2}$ Department of Mecanical Engineering, University of Sciences and Technology of Oran MB, Algeria \\ ${ }^{3}$ Department of AutomaticEngineering, University of Sciences and Technology of Oran MB, Algeria \\ ${ }^{4}$ Department of Maritime and Mechanical Engineering, Liverpool John Moore's University, United Kingdom
}

\begin{abstract}
Article Info
Article history:

Received Dec 30, 2019

Revised May 20, 2020

Accepted Jun 1, 2020

\section{Keywords:}

Airplane

LQR control

PID control

Tilt rotor

Transition

UAV

VTOL

ABSTRACT

The main thematic of this paper is controlling the main manoeuvers of a tilt rotor UAV airplane in several modes such as vertical takeoff and landing, longitudinal translation and the most important phase which deal with the transition from the helicopter mode to the airplane mode and visversa based on a new actuators combination technique for specially the yaw motion with not referring to rotor speed control strategy which is used in controlling the attitude of a huge number of vehicles nowadays. This new actuator combination is inspired from that the transient response of a trirotor using tilting motion dynamics provides a faster response than using rotor speed dynamics. In the literature, a lot of control technics are used for stabilizing and guarantee the necessary manoeuvers for executing such task, a multiple Attitude and Altitude PID controllers were chosen for a simple linear model of our tilt rotor airplane in order to fulfill the desired trajectory, for reasons of complexity of our model the multiple PID controller doesnt take into consideration all the coupling that exists between the degrees of freedom in our model, so an LQR controller is adopted for more feasible solution of complex manoeuvering, the both controllers need linearization of the model for an easy implementation.
\end{abstract}

Copyright $@ 2020$ Institute of Advanced Engineering and Science. All rights reserved.

Corresponding Author:

Aoued Houari,

Aeronautics and Propulsive Systems Laboratory,

University of Science and Technology of Oran-MB,

B. P 1505, ElMnaouer, Oran-ALgeria.

Email : houari.aoued@univ-usto.dz

\section{INTRODUCTION}

Several applications are performed nowadays by using UAVs such as rescue operations, detection and surveillance. Many UAVs configurations are established in order to ensure some objectives like trajectory planification discussed in [1]. In this paper is studied to find an optimal trajectory tracking control of a tri tilt-rotor using an LQR controller. A new model or mathematical presentation for controlling a tilt rotor is the aim subject in [2]. A reconfigurable tri tilt rotor UAV is designed in [3] for autonomous transition between the VTOL and the fixed wing flight modes by employing the direct longitudinal actuation techniques. In [4] a development of a quad rotor having a tilt wing mechanism using an LQR and sliding mode controller for settling the attitude and the altitude are presented via simulations. A proposed designs in [5] incorporates advantageous structural features which enhances the maneuverability of the rotorcraft, some new technics are developed specially for hovering control such a nonlinear state feedback controller which is proved by numerical simulations in [6], thrust vectoring technique with the highest level of flexibility, maneuverability and minimum requirement of power is discussed in [7], using a backstepping technique to achieve mode transition control of the aircraft was the subject of [8] for the ability of high speed forward flight. Other paper researches are dealed with experimental implementation of control technics that 
what we see in [9] which present a design and verification of a hybrid vertical takeoff and landing UAV, other technics of trajectory planification and path-following guidance such the model predictive control is discussed in [10] aiming to generate references for a low level attitude controller for tracking a precompiled trajectory.

A longer flight time is one of the most constraints that has to been fulfilled by an UAV, complexity of design and mathematical model, size and cost must be respected for any UAV project [11], for that the trirotor is the ideal solution for various project and missions [12]. Due to unpaired rotor a yawing moment is generated by the reaction torque, for solving this problem we propose in our paper to use the tilting angles of the two front motors differentially. In [13] a new method for controlling tri rotor-type unmanned aerial vehicles (UAV) adapted from the SE (3) nonlinear geometric method for quadrotor-type UAV. In order to ensure a good flexibility, adaptability and better control effect,the authors of [14] had used a genetic algorithm for optimizing PID parameters a PID controller is designed for a decoupled MIMO system using Kharitinov's theorem for tuning PID parametrs in [15]. Ahmad et al., [16] try to compare the performance of similtaneous perturbations stochastic approximation (SPSA) based methods. In order to tune the PID controller a method is developed in[17] based on adaptive safe experimentation dynamics (ASED). In [18] a compact tricopter configuration tilt-rotor unmanned aerial vehicle with full modes of flight from the rotor mode to the fixed wing mode and vice versa. For enabling an intelligent selection of control switch, a Fuzzy Logic Sliding Mode Controller is adopted for a Tiltrotor aircraft; an experimental verification of reliability for this controller is discussed in $[19,20]$. In section two we will give an overview on the design of our tri tilt-rotor, control strategies will be detailed in section four to controlling attitude and translational motion for our tilt-rotor using the mathematical representation discussed in section three.

\section{TRI-ROTOR AIRPLANE DESCRIPTION}

The tri tilt-rotor is in $\mathrm{T}$ form like depicted in Figure 1 is composed of two front motors $\mathrm{M}_{\mathrm{FR}}, \mathrm{M}_{\mathrm{FL}}$ and a third motor in the rear $\mathrm{M}_{\mathrm{B}}$. Our trirotor must be able to take-off vertically and transit to conventional flight and be able to return to hover mode for landing. The VTOL motion is established by vertically adjust the thrust of the three rotors in order to fulfill the desired altitude after compensating the gravity effect.

The longitudinal motion is generated by tilting the front motors to the horizontal plane (until the motors vertical axis reach the longitudinal body axis) with decreasing the rotation speed of the rear motor, while reaching the airplane mode the rear motor is totally stopped. The roll motion is controlled by making a thrust difference between the two front motors; otherwise the rear motor is used to stabilize the pitch motion in the transition phases from helicopter mode to aircraft mode and vice versa by compensating the force generated by the front motors, the yaw motion is controlled by tilting the two front motors in different direction with the same angle so we can generate a torque about the yaw axis.

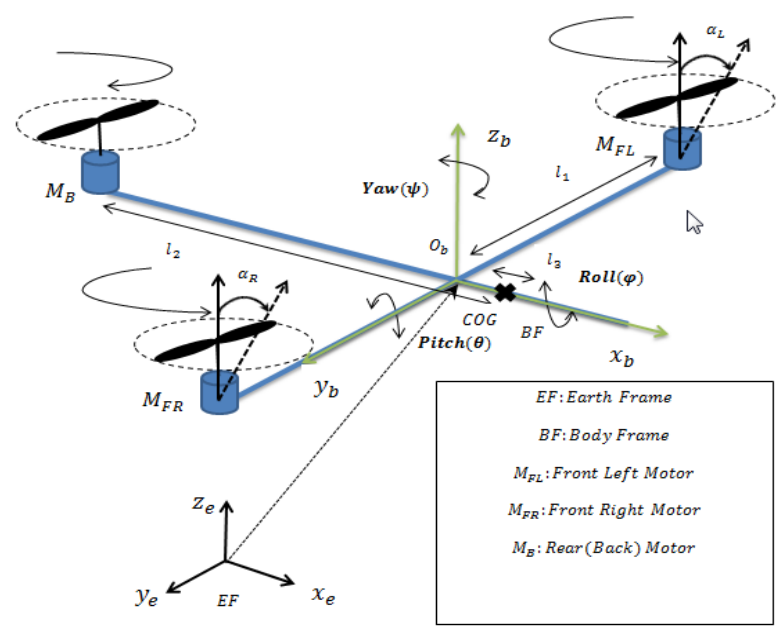

Figure 1. Tri tilt-rotor configuration

\section{MATHEMATICAL MODELING}

Generally, all moving objects in space are referred by two frames, one fixed on the body of the object $(\mathrm{BF})$ and the second named earth frame (EF) like depicted in Figure 1. To be able to design 
a control strategy, a simplified mathematical representation of the tri-rotor is needed. Due to the fact that some states are measured in (BF) while some others are measured in (EF) a frame transformation matrix $\mathrm{T}$ will be used to ensure transformation between frames.

$$
T=\left[\begin{array}{ccc}
C \psi C \phi & S \psi C \phi & S \phi \\
C \psi S \phi S \theta-S \psi C \theta & S \psi S \phi S \theta+C \psi C \theta & C \phi S \theta \\
C \psi S \phi C \theta+S \psi S \theta & S \psi S \phi C \theta-C \psi S \theta & C \phi C \theta
\end{array}\right]
$$

Where the abbreviations $C \alpha, \operatorname{S} \alpha$ are used instead of $\operatorname{Cos} \alpha$, $\operatorname{Sin} \alpha$.To develop the dynamic model of the tri tilt-rotor we consider that the structure of our UAV is rigid [17], the dynamic of the actuators is neglected assuming that we have very fast actuators [7]. Due to low velocity the drag effect is assumed negligible for both lateral and longitudinal motions. Using Newton-Euler formalism we can represent the equations of motion in body fixed frame (BF) as follows [18]:

$$
\begin{aligned}
& \sum F=m \ddot{P}_{b}+\dot{\Omega}_{b} \times\left(m \dot{P}_{b}\right) \\
& \sum M=J \ddot{\Omega}_{b}+\dot{\Omega}_{b} \times\left(J \dot{\Omega}_{b}\right)
\end{aligned}
$$

With: the Euler angle of the tilt rotor is defined as $\Omega_{b}=[\theta, \phi, \psi]$, where $\theta$ the pitch angle defined around the $\mathrm{y}_{\mathrm{b}}$ axis, $\phi$ the roll angle defined around the $\mathrm{x}_{\mathrm{b}}$ axis and $\psi$ the yaw angle defined around the $\mathrm{z}_{\mathrm{b}}$ axis, the position of the tilt rotor according to the earth frame is defined as $\mathrm{P}_{\mathrm{e}}=[\mathrm{x}, \mathrm{y}, \mathrm{z}], F=\left[F_{x b} F_{y b} F_{z b}\right]^{T}$ the external force acted on the tilt rotor, $M=\left[M_{x b} M_{y b} M_{z b}\right]^{T}$ the rotational torque of the tilt rotor, $\mathrm{m}$ the total mass of the body and $J=\left[J_{x} J_{y} J_{z}\right]^{T}$ is the moment of inertia of the body.

The external forces and the rotational torques acted on the titlrotor expressed in body frame are depicted in the equation above [21]:

$$
\begin{aligned}
& F=\left[\begin{array}{lll}
F_{L} \cos \alpha_{L}+F_{R} \cos \alpha_{R} & 0 & F_{L} \sin \alpha_{L}+F_{R} \sin \alpha_{R}+F_{B}
\end{array}\right]^{T} \\
& M=\left[\begin{array}{c}
l_{1}\left(F_{L} \cos \alpha_{L}-F_{R} \cos \alpha_{R}\right) \\
l_{1}\left(F_{L} \cos \alpha_{L}+F_{R} \cos \alpha_{R}\right)-l_{2} F_{B} \\
l_{3}\left(F_{L} \sin \alpha_{L}-F_{R} \sin \alpha_{R}\right)
\end{array}\right]
\end{aligned}
$$

with: $\alpha_{L}, \alpha_{R}$ the tilt angles of the left and the right front motors respectively. Since there are no forces acted along the $y_{b}$ axis in the body frame, the motion along this axis will not be considered.

The final equations of motion according to Newton-Euler formalism will be:

$$
\begin{aligned}
\ddot{x} & =(\cos \psi \sin \theta \cos \phi+\sin \psi \cos \phi) \frac{U_{x}}{m} \\
\ddot{z} & =(\cos \theta \cos \phi) \frac{U_{z}}{m}-g \\
\ddot{\theta} & =\left(\frac{J_{x}-J_{z}}{J_{y}}\right) \dot{\phi} \dot{\psi}+\frac{U_{\theta}}{J_{y}} \\
\ddot{\phi} & =\left(\frac{J_{z}-J_{y}}{J_{x}}\right) \dot{\theta} \dot{\psi}+\frac{U_{\phi}}{J_{x}} \\
\ddot{\psi} & =\left(\frac{J_{y}-J_{x}}{J_{z}}\right) \dot{\theta} \dot{\phi}+\frac{U_{\psi}}{J_{z}}
\end{aligned}
$$

$$
\text { With: }\left[\begin{array}{c}
U_{x} \\
U_{z} \\
U_{\theta} \\
U_{\phi} \\
U_{\psi}
\end{array}\right]=\left[\begin{array}{c}
F_{L} \cos \alpha_{L}+F_{R} \cos \alpha_{R} \\
F_{L} \sin \alpha_{L}+F_{R} \sin \alpha_{R}+F_{B} \\
l_{1}\left(F_{L} \cos \alpha_{L}+F_{R} \cos \alpha_{R}\right)-l_{2} F_{B} \\
l_{2}\left(F_{L} \cos \alpha_{L}-F_{R} \cos \alpha_{R}\right) \\
l_{3}\left(F_{L} \sin \alpha_{L}-F_{R} \sin \alpha_{R}\right)
\end{array}\right]
$$


The force generated by each motor is proportional with its supply voltage $\mathrm{F}=\mathrm{k}_{\mathrm{t}} * \mathrm{~V}$ with $\mathrm{k}_{\mathrm{t}}$ the lift coefficient, using this assumption we can write the new virtual control like in equation below:

$$
\left[\begin{array}{c}
U_{x} \\
U_{z} \\
U_{\theta} \\
U_{\phi} \\
U_{\psi}
\end{array}\right]=\left[\begin{array}{c}
k_{t f} V_{L} \cos \alpha_{L}+k_{t f} V_{R} \cos \alpha_{R} \\
k_{t f} V_{L} \sin \alpha_{L}+k_{t f} V_{R} \sin \alpha_{R}+k_{t r} V_{B} \\
l_{1}\left(k_{t f} V_{L} \cos \alpha_{L}+k_{t f} V_{R} \cos \alpha_{R}\right)-l_{2} k_{t r} V_{B} \\
l_{2}\left(k_{t f} V_{L} \cos \alpha_{L}-k_{t f} V_{R} \cos \alpha_{R}\right) \\
l_{3}\left(k_{t f} V_{L} \sin \alpha_{L}-k_{t f} V_{R} \sin \alpha_{R}\right)
\end{array}\right]
$$

\section{CONTROL STRATEGIES}

In literature some parameters have to been verified and limited in acceptable ranges including overshoot, response time and control precision, the recommended acceptable range for the overshoot is set to not exceed $10 \%$ and the control precision to not exceed $\pm 1 \%$ [19], the response time is depend on the size of the UAV and the quality of actuators used. Due to its simplicity a multiple PID's were adopted for stabilizing the UAV in hover mode for indoor performing, the role of this controller is to minimize a cost function by adjusting the input value in order to reduce the error between the desired and the measured values [19].

The dynamic model presented in previous section will be transformed into a linear state space model assuming that for a small tilting angle $\cos (\alpha)=1$ and $\sin (\alpha)=\alpha$ and assuming that the motors will be running at a voltage near their hover voltage $V_{\text {hov }}[18]$, with a chosen state and control vector:

$$
\begin{aligned}
& X=\left[\begin{array}{llllllllll}
\varphi & \dot{\varphi} & \theta & \dot{\theta} & Z & \dot{Z} & \psi & \dot{\psi} & X & \dot{X}
\end{array}\right]^{T} ; U_{a}=\left[\begin{array}{lllll}
V_{L} & V_{R} & V_{B} & \alpha_{L} & \alpha_{R}
\end{array}\right]^{T} \\
& \left\{\begin{array}{c}
\dot{X}=A X+B U_{a} \\
Y=C X+D U_{a}
\end{array}\right.
\end{aligned}
$$

with:

$$
\begin{aligned}
A & =\left[\begin{array}{llllllllll}
0 & 1 & 0 & 0 & 0 & 0 & 0 & 0 & 0 & 0 \\
0 & 0 & 0 & 0 & 0 & 0 & 0 & 0 & 0 & 0 \\
0 & 0 & 0 & 1 & 0 & 0 & 0 & 0 & 0 & 0 \\
0 & 0 & 0 & 0 & 0 & 0 & 0 & 0 & 0 & 0 \\
0 & 0 & 0 & 0 & 0 & 1 & 0 & 0 & 0 & 0 \\
0 & 0 & 0 & 0 & 0 & 0 & 0 & 0 & 0 & 0 \\
0 & 0 & 0 & 0 & 0 & 0 & 0 & 1 & 0 & 0 \\
0 & 0 & 0 & 0 & 0 & 0 & 0 & 0 & 0 & 0 \\
0 & 0 & 0 & 0 & 0 & 0 & 0 & 0 & 0 & 1 \\
0 & 0 & 0 & 0 & 0 & 0 & 0 & 0 & 0 & 0
\end{array}\right] ; B=\left[\begin{array}{cccccc}
0 & 0 & 0 & 0 & 0 \\
\frac{l_{3} K_{t f}}{J_{x}} & -\frac{l_{3} K_{t f}}{J_{x}} & 0 & 0 & 0 \\
0 & 0 & 0 & 0 & 0 \\
\frac{l_{1} K_{t f}}{J_{y}} & \frac{l_{1} K_{t f}}{J_{y}} & \frac{-l_{2} K_{t b}}{J_{y}} & 0 & 0 \\
0 & 0 & 0 & 0 & 0 \\
\frac{K_{t f}}{m} & \frac{K_{t f}}{m} & \frac{K_{t b}}{m} & 0 & 0 \\
0 & 0 & 0 & 0 & 0 \\
0 & 0 & 0 & \frac{l_{3} K_{t f} V_{\text {hov }}}{J_{z}} & -\frac{l_{3} K_{t f} V_{\text {hov }}}{J_{z}} \\
0 & 0 & 0 & 0 & 0 \\
0 & 0 & 0 & \frac{K_{t f} V_{\text {hov }}}{m} & \frac{K_{t f} V_{\text {hov }}}{m}
\end{array}\right] \\
C & =\left[\begin{array}{llllllllll}
1 & 0 & 0 & 0 & 0 & 0 & 0 & 0 & 0 & 0 \\
0 & 0 & 1 & 0 & 0 & 0 & 0 & 0 & 0 & 0 \\
0 & 0 & 0 & 0 & 1 & 0 & 0 & 0 & 0 & 0 \\
0 & 0 & 0 & 0 & 0 & 0 & 1 & 0 & 0 & 0 \\
0 & 0 & 0 & 0 & 0 & 0 & 0 & 0 & 1 & 0
\end{array}\right] ; D=0 .
\end{aligned}
$$

All the parameters used during the dynamic modeling are cited in the Table 1:

Table 1. Parameters of Tilt rotor

\begin{tabular}{cc}
\hline Parameters & Values \\
\hline$l_{3}$ & $0.05 \mathrm{~m}$ \\
$l_{2}$ & $1.2 \mathrm{~m}$ \\
$l_{1}$ & $0.5 \mathrm{~m}$ \\
$k_{t f}$ & 0.7 \\
$k_{t b}$ & 0.5 \\
$J_{x}$ & $0.1946 \mathrm{~kg} . \mathrm{m}^{2}$ \\
$J_{y}$ & $0.1271 \mathrm{~kg} \mathrm{~m}^{2}$ \\
$J_{z}$ & $0.2593 \mathrm{~kg} . \mathrm{m}^{2}$ \\
$m$ & $2.5 \mathrm{~kg}$ \\
$l_{3}$ & $0.05 \mathrm{~m}$ \\
\hline
\end{tabular}


Using this state space representation, a PID parallel structure formulated by the equation below is used for controlling our UAV:

$$
u(t)=k_{p} e(t)+k_{i} \int_{0}^{t} e(t) d t+k_{d} \dot{e}(t)
$$

with: $k_{p} ; k_{i}$ and $k_{d}$ proportional, integral and derivative gains respectively and $e(t)$ is the error between the desired and the measured values.The PID parameters will be tuned using Ziegler Nichols technique as follows [22, 23]:

- We have to use only proportional feedback control.

- Reduce the integrator and derivative gains to zero.

- Increase $k_{p}$ from zero to some critical value $k_{p}=k_{c r}$ at which oscillations occur.

- Note the value $k_{c r}$ and the corresponding period of sustained oscillations, $P_{c r}$.

The controller gains are calculated like in Table 2. By applying the Ziegler Nichols technique cited above and according to Table 2 the PIDs parameters for each channel are calculated and mentionned in Table 3. Aiming to perform a vertical take-off motion at $(\mathrm{Z}=2 \mathrm{~m})$ with an equilibrium point $(\psi=\phi=\theta=$ $\left.0 \mathrm{rad} ; \mathrm{X}=0 \mathrm{~m} ; \mathrm{V}_{\text {hov }}=10\right)$, the simulation result of the PID controller are depicted in Figure 2.

Table 2. Ziegler nichols PID gains calculation

\begin{tabular}{cccc}
\hline PID Types & $\mathrm{k}_{\mathrm{p}}$ & $T_{\mathrm{i}}=k_{p} / k_{i}$ & $T_{\mathrm{d}}=k_{d} / k_{p}$ \\
\hline P & $0.5 k_{c r}$ & $\infty$ & 0 \\
PI & $0.45 k_{c r}$ & $P_{c r} / 1.2$ & 0 \\
PID & $0.6 k_{c r}$ & $P_{c r} / 2$ & $P_{c r} / 2$ \\
\hline
\end{tabular}

Table 3. PID parameters for each channel

\begin{tabular}{cccc}
\hline PID Types & $\mathrm{k}_{\mathrm{p}}$ & $k_{i}$ & $k_{d}$ \\
\hline Roll & 0.036 & 0.001 & 0.189 \\
Pitch & 0.036 & 0.0017 & 0.192 \\
Yaw & 0.048 & 0.002 & 0.252 \\
Altitude & 22.21 & 7.19 & 16.84 \\
Longitudinal & 2.87 & 0.33 & 6.06 \\
\hline
\end{tabular}
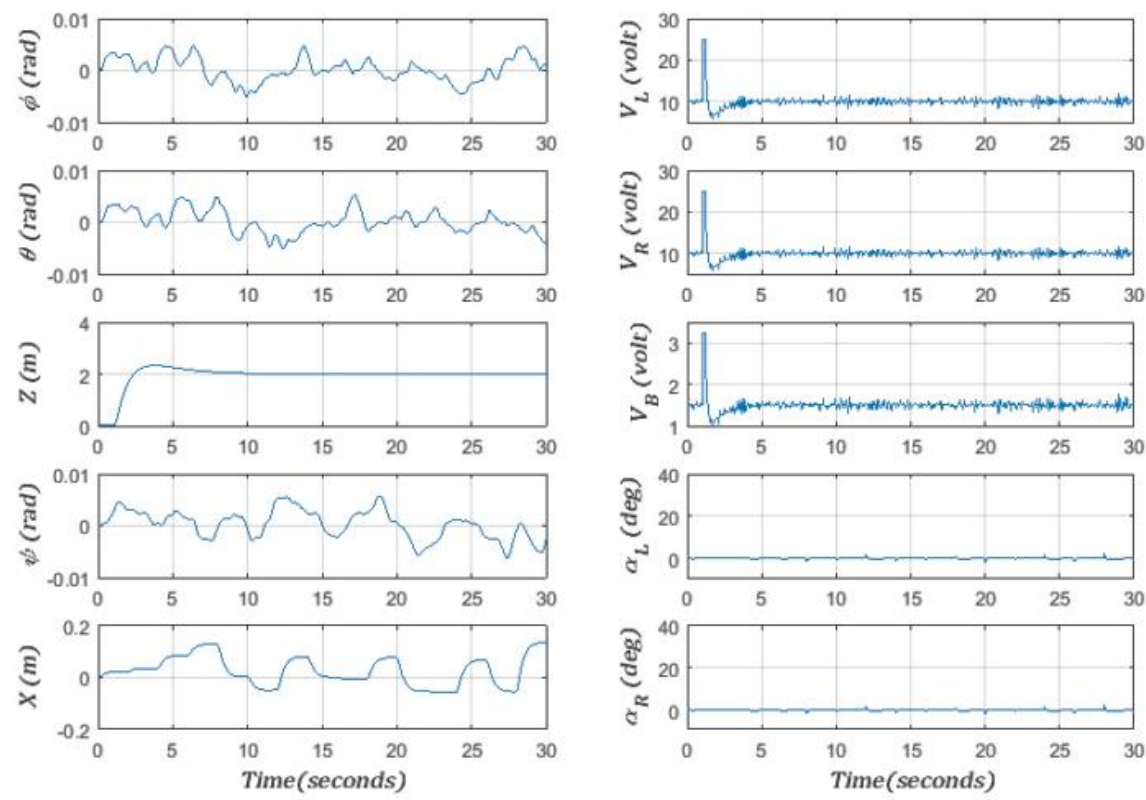

Figure 2. Vertical take-off motion PID outputs (left), Vertical take-off motion PID inputs (right) $\left(\mathrm{Z}=2 \mathrm{~m} ; \psi=\phi=\theta=0 \mathrm{rad} ; \mathrm{X}=0 \mathrm{~m} ; \mathrm{V}_{\text {hov }}=10\right)$ 
From Figure 2(Left), we can resume that our PID is performing good against the altitude reference changes taking into account an overshoot of $17 \%$ which don't allow our UAV to perform an indoor reference tracking, the response time is about $8.5 \mathrm{sec}$. The two front motors have a hovering voltage of $\mathrm{V}_{\text {hov }}=10$ volt and can attain 25 volts maximum so regarding to Figure 2(Right), the PID generate the necessary voltage for the desired motion respecting the maximum value and return to hovering voltage after the steady state is achieved whereas the rear motor is used just for stabilizing the pitch moment and its contribution in VTOL motion is minim, the maximum supplied voltage depend on the location where is placed according to the center of gravity of the Tiltrotor(the rear motor is placed far from the center of gravity, in the figure above the rear motor voltage attain 3.3 volts for stabilizing the altitude which is lower than the two front motors because of their location(there are near the center of gravity)).

In a second test, we try to perform a longitudinal motion of ( $X=4 \mathrm{~m})$ at an equilibrium point $\left(\psi=\phi=\theta=0 \mathrm{rad} ; \mathrm{Z}=2 \mathrm{~m} ; \mathrm{V}_{\mathrm{hov}}=10\right)$ like illustrate in Figure 3. From Figure 3, our UAV will have the same problem for indoor reference tracking for longitudinal motion because there is an overshoot of $10 \%$; we notice also that our UAV in the longitudinal motion is quick comparing to the vertical take-off motion with a response time about $6 \mathrm{sec}$. The two motion performed above indicate that the PID controllers are not a good controller for indoor reference tracking according to the simulation in Figures 2 and 3 because of the strong coupling of the UAV system dynamics, in order to solve this problem, we have to choose another controller that takes into account all the coupling effects of the UAV dynamics, the most well-known regulator for linear systems which can perform well for indoor motion, minimizing the cost function and give us more feasible solution of this problem is a the LQR controller. Using a single LQR controller will help us to feedback all the states in one step (based on matrix calculus) to select the desired output however in PID controller we have used a multiple PID's because that the PID can cover just one channel (based on scalar calculus) so five PID's are used for comparing all the outputs with the outputs generated from LQR controller.
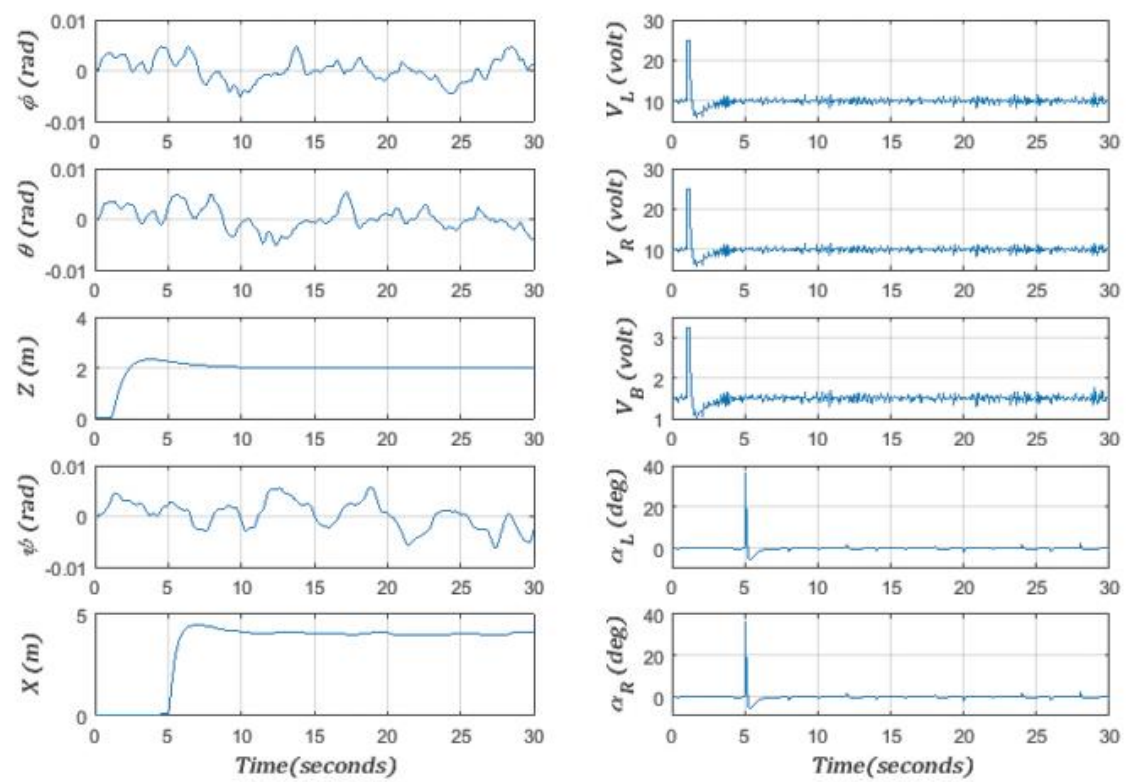

Figure 3. Longitudinal motion PID outputs (left), longitudinal motion PID inputs (right)

$$
\left(\mathrm{Z}=2 \mathrm{~m} ; \psi=\phi=\theta=0 \mathrm{rad} ; \mathrm{X}=4 \mathrm{~m} ; \mathrm{V}_{\text {hov }}=10\right)
$$

The LQR controller is used to obtain the best control sequence that minimizes the cost function detailed in the equation below [14, 24, and 25], using the state space representation in (12):

$$
J=\int_{0}^{\infty} X(t)^{T} Q X(t)+U(t)^{T} R U(t)
$$

where $\mathrm{R}$ is a positive definite weighting matrix and $\mathrm{Q}$ is a weighting matrix that can be positive semi-definite, $\mathrm{U}(\mathrm{t})$ represents the optimal control law detailed in the equation above: 


$$
U(t)=-K X(t)=-R^{-1} B^{T} P X(t)
$$

$K$ is the linear optimal feedback gain and $P$ is the solution of Riccati equation represented by (17):

$$
P A+A^{T}-P B R^{-1} P+Q=0
$$

The two weighting matrices have to be tuned manually based on the priority or weighting of each state $(\mathrm{Q}$ matrix) and input( $\mathrm{R}$ matrix) that I have fixed in order to find a compromise between the steady state performance and the actuators energy applied respecting the parameters limit ranges discussed above. Results of the LQR simulation for a vertical take-off $(\mathrm{Z}=2 \mathrm{~m})$ with the same operating point used previously for the PID controller are presented in Figure 4.
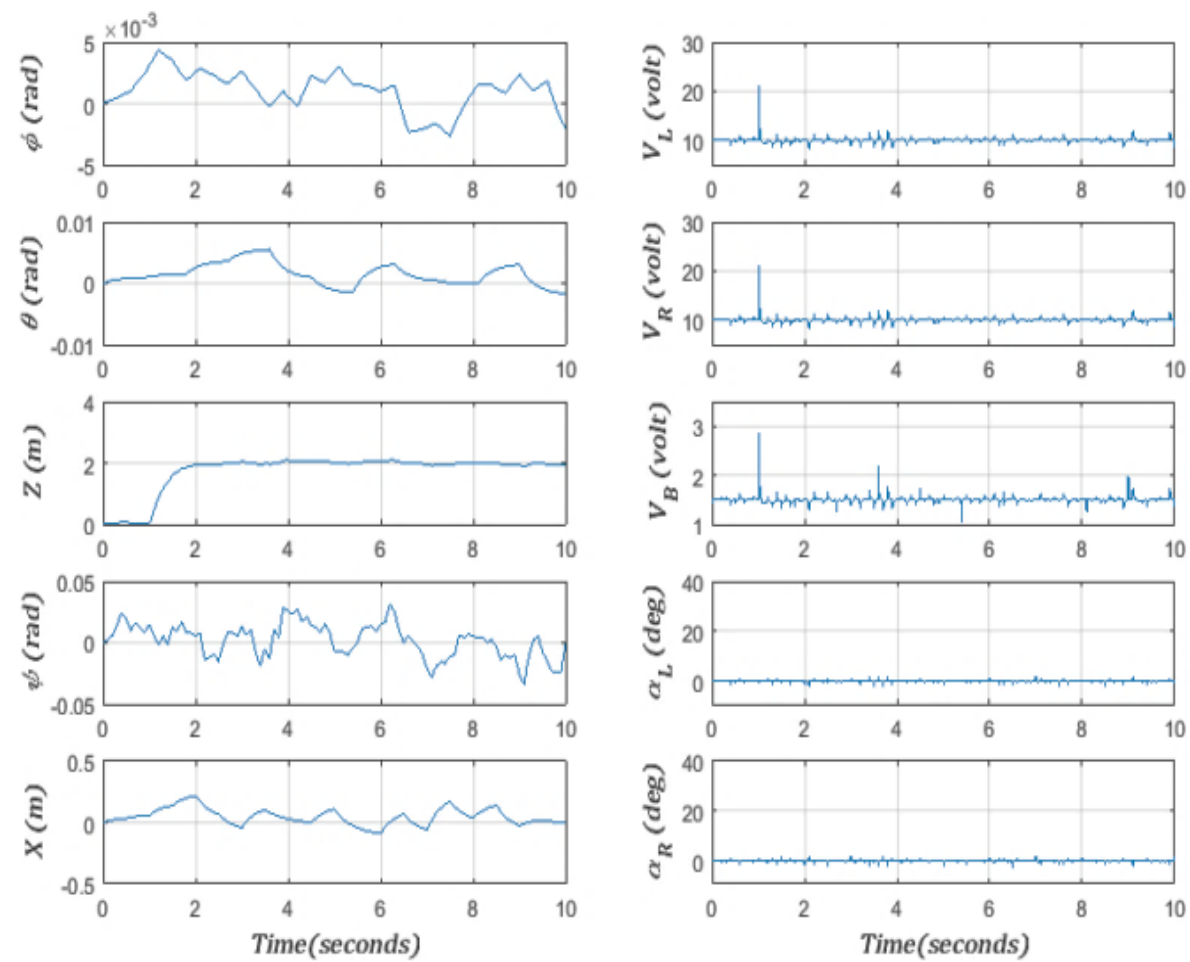

Figure 4. Vertical Take-Off motion LQR outputs (left), vertical Take-Off motion LQR inputs (right) $\left(\mathrm{Z}=2 \mathrm{~m} ; \psi=\phi=\theta=0 \mathrm{rad} ; \mathrm{X}=0 \mathrm{~m} ; \mathrm{V}_{\mathrm{hov}}=10\right)$

It can be seen in Figure 4 that the overshoot is reduced to $0 \%$ and the reponse time to $3 \mathrm{sec}$ instead of $17 \%$ and $8.5 \mathrm{sec}$ for the PID controller, from this result we can conclude that the LQR controller is more powerful than the PID for indoor performing. Some perturbation illustrates in Figure 4 for the rest of outputs but with no significant effect on the desired motion, these small perturbations highlight the strong coupling dynamics of the UAV and prove the capability of the LQR to take into account all the constraints attached to the dynamics complexity.

For the longitudinal motion with a desired value of $(X=4 \mathrm{~m})$ and a specific operating point $(\psi=\phi=\theta=0$ rad; $Z=2 \mathrm{~m})$, the $\mathrm{LQR}$ controller simulation results are given in Figure 5. Comparing to the response characteristics obtained in PID controller for longitudinal motion, the use of the LQR controller is more feasible such the performances criterion discussed above are satisfied for an overshoot of $0 \%$ and a time response of $3 \mathrm{sec}$.the yaw motion is affected by the longitudinal motion because there is a strong coupling between this two degrees of freedom such the two motions are performed by tilting the two front motors, the actuators behavior for the LQR controller is illustrated in Figure 5 and we can remark clearly that comparing to the PID actuators control signals, the LQR act less aggressive which give us very good control margin. We conclude this paper by a performance indexes comparison for both controllers which is illustrated in Table 4. 

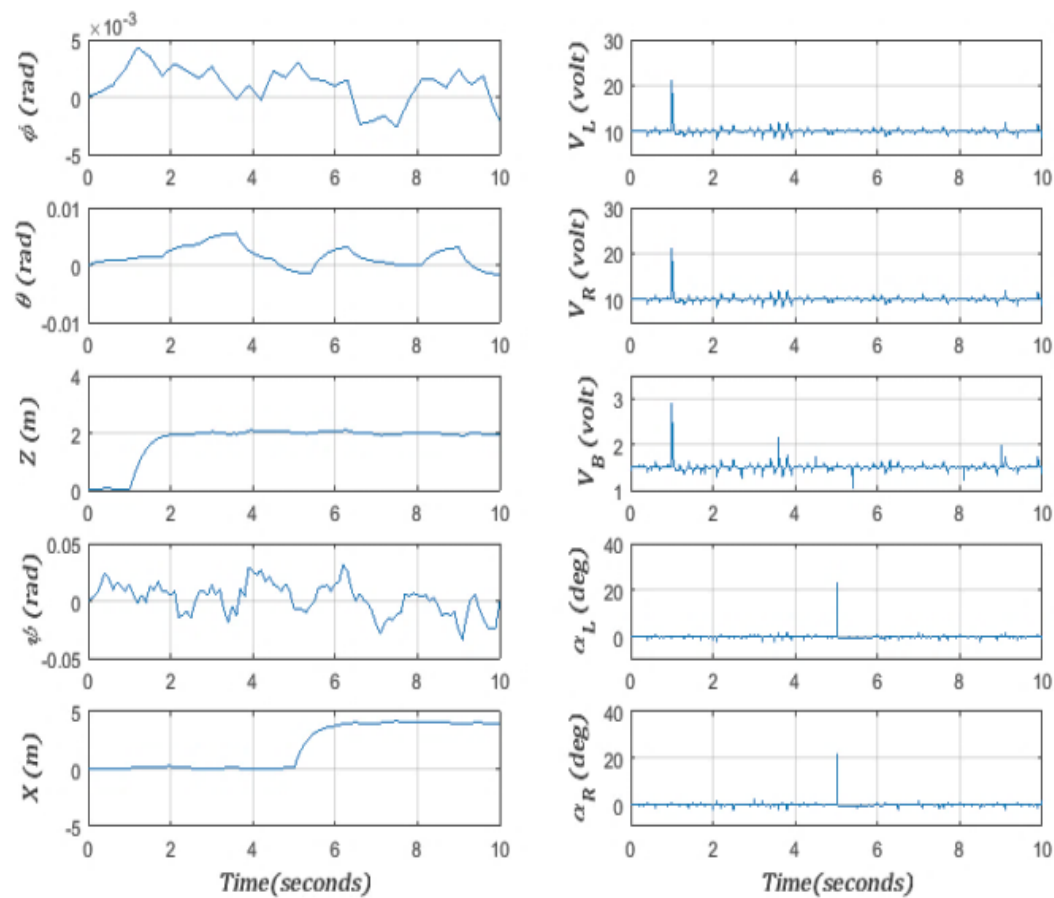

Figure 5. Longitudinal motion LQR outputs (feft), longitudinal motion LQR inputs (right) $\left(\mathrm{Z}=2 \mathrm{~m} ; \psi=\phi=\theta=0 \mathrm{rad} ; \mathrm{X}=4 \mathrm{~m} ; \mathrm{V}_{\mathrm{hov}}=10\right)$

Table 4. PID and LQR performance indexes comparison

\begin{tabular}{lcccc}
\hline \multicolumn{1}{c}{ PID Types } & PID VTOL & LQR VTOL & PID Longitudinal & LQR Longitudinal \\
\hline Overshoot (\%) & 17 & 0 & 10 & 0 \\
Response Time (sec) & 8.5 & 3 & 6 & 3 \\
Control precision $(\%)$ & 0.016 & 0.001 & 0.012 & 0.001 \\
\hline
\end{tabular}

\section{CONCLUSION}

In this paper we have developed a dynamic mathematical model for a tilt rotor airplane, aiming to perform a vertical take-off and longitudinal motions, two control technics are proposed and applied on a linear dynamics model. The simulation results of both of the controllers were compared using some performance indexes such as the overshoot, response time and control precision, the LQR controller was more powerful comparing to PID controller in all performance indexes for both vertical take-off and longitudinal motions.

\section{REFERENCES}

[1] C. Papachristos, K. Alexis, A. Tzes., "Linear Quadratic Optimal Trajectory-Tracking Control of a Longitudinal Thrust Vectoring-Enabled Unmanned Tri-Tilt Rotor," Thirty-Nine Annual IEEE Conference Industrial Electronics Society, pp. 4174-4179, 2013.

[2] D. Anh Ta, I. Fantoni, R. Lozano., "Modeling and Control of a Tilt tri-rotor Airplane," American Control Conference, pp. 131-136, 2012.

[3] C. Papachristos, K. Alexis, A. Tzes., "Trajectory Control of an Unmanned Tri-Tilt Rotor in Hover Flight via Direct Longitudinal Actuation," Twenty-First Mediterranean Conference in Control and Automation, pp. 369-374, 2013.

[4] K.T. Oner, E. Cetinsoy, E. Sirimoglu, C. Hancer, T. Ayken, and M. Unel., "LQR and SMC Stabilization of a New Unmanned Aerial Vehicle," World Academy of Science, Engineering and Technology, pp. 554-559, 2009.

[5] J. Escarefio, A. Sanchez, O. Garcia and R. Lozano., "Triple Tilting Rotor mini-UAV: Modeling and Embedded Control of the Attitude," American Control Conference, pp. 3476-3481, 2008.

[6] L. Feng, Z. William, D.B. Robert., "Robust Hovering Control of a PVTOL Aircraft," IEEE Transaction on Control Systems Technology, vol. 7, no. 3, pp. 343-351,1999.

[7] M. Kara, A.Lanzon., "Design and Control of Novel Tri-rotor UAV," Proceedings of 2012 UKACC International Conference on Control, pp. 304-309, 2012. 
[8] P. Fan, X. Wang, K.Y. Kai., "Design and Control of a Tri-rotor Aircraft," International conference on Control and Automation, pp. 1972-1979, 2010.

[9] H. Gu, X. Lyu, Z. Li, S. Shen and F. Zhang., "Development and Experimental Verification of a Hybrid vertical Take-off and Landing(VTOL) Unmanned Aerial vehicle(UAV)," International Conference on Unmanned Aircraft Systems, pp. 160-169, 2017.

[10] G. Francisco, V. Rafael, F. C. Eduardo., "An iterative Model Predictive control Algorithm for UAV Guidance," IEEE Transactions on Aerospace and Electronic Systems, vol. 51, no. 3, pp. 2406-2419, 2015.

[11] S. Yoon, S. J. Lee, B. Lee, C. J. Kim, Y. J. Lee and S. Sung, "Design and Flight Test of a Small TriRotor Unmanned Vehicle with a LQR Based Onboard Attitude Control System," International Journal of Innovative Computing, Information and Control, pp. 2347-2360, 2013.

[12] D. W. Yoo, H. D. Oh, D. Y. Won and M. J. Tahk, "Dynamic Modeling and Stabilization Techniques for Tri-Rotor Unmanned Aerial Vehicles," International Journal of Aeronautical and Space Science, vol. 11, no. 3, pp. 167-174, 2010.

[13] D. T. Huang, T. H. H. Le and N. H. Nguyen, "Adapting SE (3) Nonlinear Geometric Method to Control Single-Tri Rotor with Integrator," American Journal of Aerospace Engineering, pp. 96-105, 2018.

[14] Y. Q. Zhang, W. P. Zhaw and S. Xiang, "Control Law and Simulation Analysis of Vertical Take-Off and Landing Stage of Tilt-Rotor Aircraft,” Journal of Advanced Materials Research, pp. 521-524, 2015.

[15] S. K. Pandey, J. Dey and S. Banerjee, "Design of Optimal PID Controller for Control of Twin Rotor MIMO System (TRMS)," Advances in Power and Control Engineering, pp. 93-106, 2020.

[16] M.A. Ahmad, S.I. Azuma and T. Sugie, "Performance analysis of model-free PID tuning of MIMO systems based on simultaneous perturbation stochastic approximation," Expert Systems with Applications, pp. 6361-6370, 2014.

[17] M.R.B. Ghazali, M.A.B. Ahmad and R. Ismail, "Adaptive safe experimentation dynamics for data-driven neuroendocrine-PID control of MIMO systems,” IETE Journal of Research, pp. 1-14, 2019.

[18] C. Chen, J. Zhang, D. Zhang and L. Shen, "Control and Flight Test of a Tiltrotor Unmanned Aerial Vehicle," International Journal of Advanced Robotic Systems, pp.1-12, 2017.

[19] C. S. Yoo, S. D. Ryu, B. J Park, Y. S Kang, C. M Elias and S. B Jung, "Actuator controller based on Fuzzy Sliding Mode Control of Tilt Rotor Unmanned Aerial Vehicles," International Journal of Control, Automation and systems, vol. 12, no. 6, pp. 1257-1265, 2014.

[20] M. Nasr, M. Ashraf, M. S Hussein, A. S Salem, C. M Elias, O. M Shehata and E. I Moragn., "A comparative study on the control of UAVs for Trajectory tracking by MPC, SMC, Backstepping, and Fuzzy Logic controllers," IEEE International Conference in Vehicular Electronics and Safety, pp. 1-6, 2018.

[21] Z. Prime, J. Sherwood, M. Smith, and A Stabile, "Remote Control Vertical Take-off and Landing Model Aircraft," Level 4 Honors Project Final Report, 2005.

[22] Ch. B. Prakash, R. S Naik, "Tuning of PID Controller by Ziegler Nichols Algorithm for Position Control of DC Motor," International journal of Innovative Science, Engineering and Technology, vol. 1, no. 3, pp. 379-382, 2014.

[23] T. R. Kurfess, "Getting in tune with Ziegler-Nichols," Academic Viewpoint column, Control Engineering magazine, 2007.

[24] K. K. Yit, P. Rajendran, and L. K Wee, "Proportional-derivative Linear Quadratic Regulator Controller Design for improved Longitudinal Motion Control of Unmanned Aerial Vehicles," International journal of Micro Air Vehicles, vol. 8, no. 1, pp. 41-50, 2016.

[25] A. M. Ayad, H. Wahid, "Optimal Tuning of Linear Quadratic Regulator controller Using a Particle Swarm Optimization for Two-Rotor Aero dynamical System," International journal of Electronics and Communication Engineering, pp. 196-202, 2017.

\section{BIOGRAPHIES OF AUTHORS}

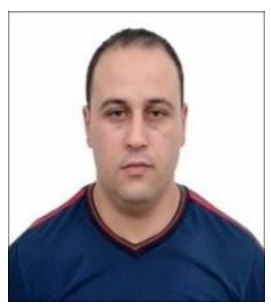

Aoued Houari Was born on 1985 in Relizane, Algeria. He received his B.S (State engineer) Engineering degree in automatism from University of Sciences and Technology of Oran (USTOMB), Algeria, in 2008. He received his M.S (Magister) degree in Electronics, Automatic, Robotic and productic option from USTO-MB University in 2012. He is currently an associate professor at UHBC university. His main research interest is within the control and applications of advanced control technics, design and control of UAV.

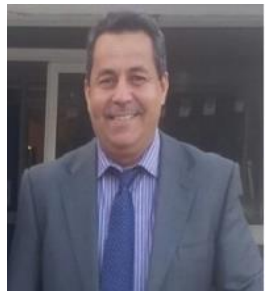

Imine Bachir was born on 1962 in Oran, Algeria. He received his B.S (State engineer) Engineering degree in mechanical engineering from University of Sciences and Technology of Oran (USTOMB), Algeria, in 1986. He received his M.S (Magister) degree in Mechanical engineering, option: Energetical from USTO-MB university in 1993. He received his PHd in Mechanical engineering, option: Energetical from USTO-MB university in 2004. He is currently an associate professor in USTO-MB University since 1987. His main research interest is UAV aerodynamic design. 


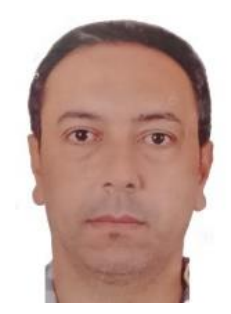

Della Krachai Mohamed was born on 1973, in Oran. In 1996, he obtained his electronics engineer diploma from the University of Science and Technology USTO-MB. After that he obtained the Magister degree in 2001, then the Doctorate degree in electronics in 2009. He is currently a researcher/lecturer at the Department of Automatics at USTO-MB University. His researches focus on robotics, control systems, and renewable energies.

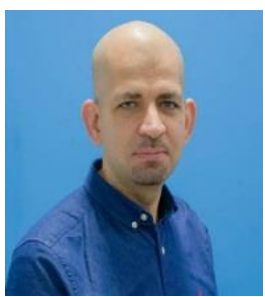

Kara Mohamed Kara is currently a Senior Lecturer in Robotics. Before joining Liverpool John Moores University, he held several academic and research positions with Birmingham City University, The University of Manchester and Coventry University. He worked also in industry as a Switching Systems Engineer at MTN Group before moving to academia. Dr Mohamed holds a PhD degree in Control Engineering from The University of Manchester. His main research interest is within the control and applications of advanced systems including autonomous robotic systems. 